\title{
PREDICTION OF EARING IN CUP DRAWING FCC MATERIALS
}

\author{
F. BARLAT, S. PANCHANADEESWARAN and O. RICHMOND \\ Alcoa Laboratories, Alcoa Center, PA 15069, USA
}

\section{INTRODUCTION}

Several attempts have been made to predict earing tendency during cup drawing from known properties of the initial sheet material. Hill ${ }^{1}$ first proposed an explanation for ear and trough positions based on his anisotropic yield function and on the assumption of no hold-down pressure (plane stress) or no thickening of the blank (plane strain). In order to take better account of the microstructure of metals, models based on the plastic slip of a single crystal or of a polycrystal have been developed. Tucker's approach ${ }^{2}$ based on the Schmid law for a FCC single crystal is well known and it is in reasonable agreement with experiments. Numerous studies have used the Taylor ${ }^{3} /$ Bishop and $\mathrm{Hill}^{4}$ polycrystal model to describe the material behavior, and some assumptions concerning the state of stress under the blank holder to predict the earing profile 5,6 . Recently, Panchanadeeswaran et al. ${ }^{7}$ proposed a simple model of earing based on the Taylor/Bishop and Hill theory. They used this model to predict the earing profile in cups of high purity aluminum processed with various amounts of cold rolling. They obtained theoretical earing profiles in good agreement with experimental ones for sheets with a high reduction. However, the agreement was not very good for sheets with a low reduction. In this work, after we first describe the model and the associated assumptions employed, we show that these predictions can be substantially improved when suitable assumptions concerning the stress and strain state under the blank holder are made.

The material is assumed to possess orthotropic symmetry with principal axes, (rolling, transverse and normal directions) denoted by $x, y$ and $z$. The plastic behavior of the material is described by the yield surface and the associated flow rule. Now, considering the cup drawing operation, we assume that ears start to form in the flange and that the final positions of ears and troughs coincide with initial positions. This assumption is supported by many experimental observations. Therefore, one needs only to analyze the state of stress and strain at the beginning of the drawing process. In other words, work-hardening and texture evolution are neglected. In addition, the effects of friction are not accounted for. If $v_{\mathrm{r}}, \mathrm{v}_{\boldsymbol{\theta}}$ and $\mathrm{v}_{\mathrm{Z}}$ represent components of the velocity vector at any point of the flange with respect to cylindrical coordinates (Fig. 1), the strain rate components can in principle be calculated and be related to the stress by the constitutive law. However, the stress state at any point of the flange is unknown and nonuniform. Therefore, two other assumptions are necessary to be able to calculate the earing profile. Although $v_{\theta}$ and $v_{z}$ are not equal to zero they are negligible when compared to the radial velocity. Moreover, it is assumed that earing tendency is mainly imposed by the radial velocity of points located at the rim of the flange. Except at the very end of the drawing operation, these points are always part of the flange. Using the 
relationship between velocity field and strain rates, we then obtain for a point on the rim7:

$$
\frac{d v_{r}}{d \theta}=2 \frac{\dot{\varepsilon}_{r \theta}}{\dot{\varepsilon}_{\theta}} \mathbf{v}_{r}
$$

Two simple extreme cases can be considered. In one case, the blank holder is supposed to impose a pressure which is negligible compared to the stress actually developed in the blank; in the other, it is supposed to maintain sufficient pressure to inhibit any thickening of the sheet. These two cases are referred to as plane stress and plane strain, respectively.

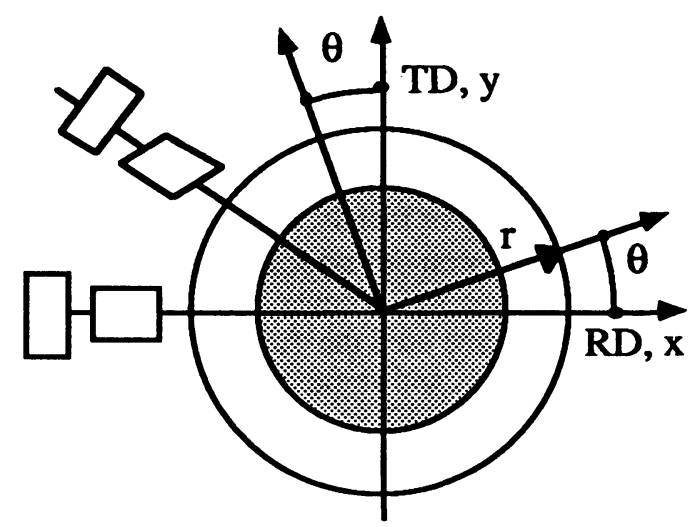

Figure 1. Cup drawing geometry and physical interpretation of earing formation by shear deformation.

Assuming yielding all around the flange, and using the normality flow rule together with tensor transformations give:

$$
\dot{\varepsilon}_{x x} \frac{d \sigma_{x x}}{d \theta}+2 \dot{\varepsilon}_{x y} \frac{d \sigma_{x y}}{d \theta}+\dot{\varepsilon}_{y y} \frac{d \sigma_{y y}}{d \theta}=0
$$

for both plane stress and plane strain. Noting that $\sigma_{\mathrm{r}}=\sigma_{\mathrm{r} \theta}=0$ at the rim of the flange and using the tensor transformation for strains then gives:

$$
\frac{1}{\sigma_{\theta}} \frac{d \sigma_{\theta}}{d \theta}=2 \frac{\dot{\varepsilon}_{r \theta}}{\dot{\varepsilon}_{\theta}}=2 \Gamma
$$

where the parameter $\Gamma$ is that introduced by BARLAT and RICHMOND $^{8}$ to characterize the shear deformation occurring during uniaxial tension (or compression) in a given sheet direction. Shear occurs if the considered direction is not a material symmetry axis; i.e., when principal stress and principal strain rate axes are not coincident. Equation 3 relates the variation of circumferential stress in various directions to the parameter $\Gamma$. Note that the stress $\sigma_{\theta}$, corresponds to the yield stress in a specimen oriented at angle, $\theta+\pi / 2$, to the rolling direction. For the plane stress case, (no hold-down pressure), it should be taken as simple yield stress; for the plane strain case (no thickening), it should be taken as plane strain yield stress. Combining Equations 1 and 3 gives: 


$$
\frac{1}{v_{r}} \frac{d v_{r}}{d \theta}=\frac{1}{\sigma_{\theta}} \frac{d \sigma_{\theta}}{d \theta}
$$

Therefore, it appears that the curve that represents the normalized variation of the radial velocity of the rim of the flange as a function of angular position is the same as the one representing the normalized yield stress variation corresponding to the appropriate hold-down conditions.

Equation 4 is in agreement with Hill's statement ${ }^{1}$ : "In both plane stress and plane strain, the tangents at the points where ears and hollows develop are in the direction for which the yield stress has a stationary value." Actually, since there is a relationship between the yield stress and the shear parameter $\Gamma$ either in uniaxial or in plane strain tension, this simple model clearly shows that this parameter is the one that actually relates earing to uniaxial or plane strain properties, and Fig. 1 illustrates the physical interpretation of this relationship. The $R$ value, width to thickness strain ratio in uniaxial tension does not naturally enter into this simple model. This does not mean that no correlation between earing profile and $R$ value can exist. Actually, Rollett et al. ${ }^{9}$ have shown such a relationship, but it implicitly assumes plane stress (no blank holder force), because the $R$ value is defined for uniaxial tension only. In the present work, Equation 4 has been shown to predict earing profiles for both plane strain and plane stress cases.

\section{APPLICATION TO SINGLE CRYSTAL}

Assuming $\{111\}<1 \overline{1} 0>$ slip, the yield surface of the FCC single crystal in the cube position associated with the Schmid law can be expressed as:

$F=\left(\left|\sigma_{y y}-\sigma_{z z}\right|+\left|\sigma_{z x}\right|+\left|\sigma_{x y}\right|\right)^{m}+\left(\left|\sigma_{z z}-\sigma_{x x}\right|+\left|\sigma_{y z}\right|+\left|\sigma_{x y}\right|^{m}+\left|\sigma_{x x}-\sigma_{y y}\right|+\left|\sigma_{z x}\right|+\left|\sigma_{y z}\right|^{m}=(\sqrt{6} \tau)^{m}\right.$

where $\tau$ is the critical resolved shear stress and $m=\infty$. For uniaxial tension (or compression) in a direction defined by the angle $\theta$, Equation. 5 gives the yield stress variation with respect to the tension direction; namely,

$$
\sigma=\frac{\sqrt{6} \tau}{\cos \theta(\cos \theta+\sin \theta)} \quad 0^{\circ}<\theta<45^{\circ}
$$

For plane strain tension (or plane strain compression), it is the more convenient to consider the plane stress shear state, which differs by a hydrostatic pressure only. For this case, the symmetry of the cubic single crystal requires that the stresses $\sigma_{x x}$ and $\sigma_{y y}$ have opposite signs as is the case for the isotropic material. Such conditions require $-\sigma_{\theta}$ to be equal to $\sigma_{\mathrm{r}}=\sigma / 2$. Restricting the angle $\theta$ to be between $0^{\circ}$ and $45^{\circ}$ only, the use of Equation. 5 leads to two expressions of $\sigma$ depending on whether the angle $\theta$ is smaller or larger than $22.5^{\circ}$. Assuming that a superimposed hydrostatic pressure does not influence the results, the major plane strain yield stress variation is given by the following relations:

$$
\sigma=\frac{\sqrt{6} \tau}{\cos 2 \theta} \quad 0^{\circ}<\theta<22.5^{\circ}, \quad \sigma=\frac{2 \sqrt{6} \tau}{\cos 2 \theta+\sin 2 \theta} \quad 22.5^{\circ}<\theta<45^{\circ}
$$

The cup height profile calculated for the cube single crystal is shown in Fig. 2 for both plane stress and plane strain conditions and these are compared with the experimental cup 
height profile measured by Tucker ${ }^{2}$. The plot indicates that the plane strain prediction is in better agreement with the experimental profile than the plane stress condition which does not even lead to the well known $0^{\circ}$ and $90^{\circ}$ earing for such a material.

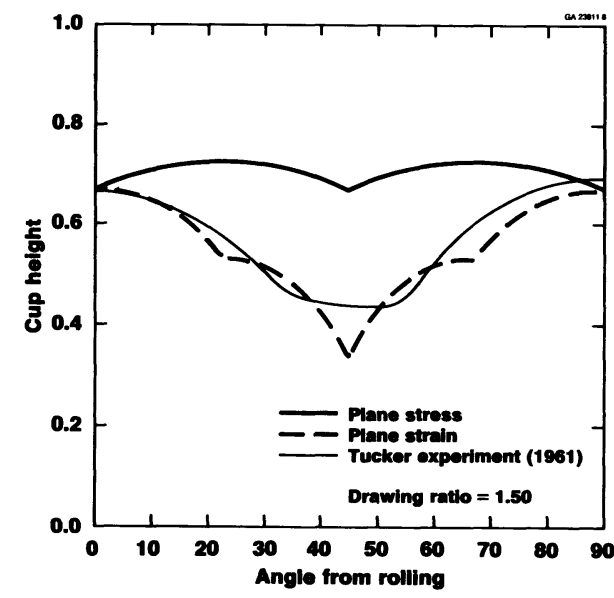

Figure 2. Predicted plane stress and plane strain and experimental cup height profiles for a single crystal in cube position

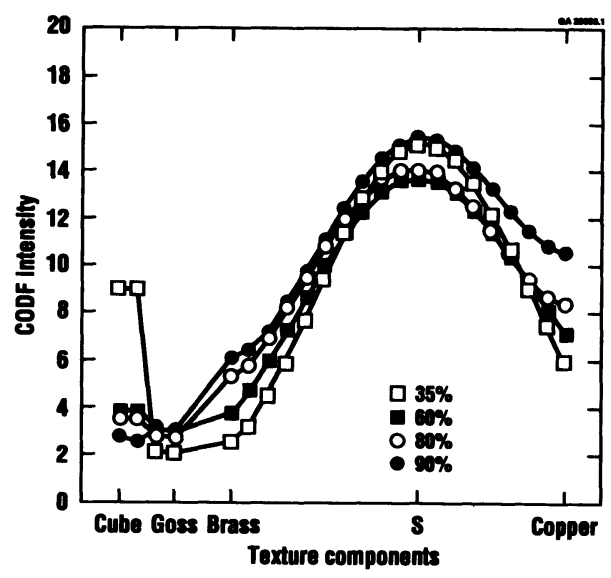

Figure 3. CODF intensity along the squeleton line for high purity aluminum sheets with various cold rolling reductions.

\section{APPLICATION TO HIGH PURITY ALUMINUM POLYCRYSTALS}

The previous analysis is applied in this section to the case of high purity aluminum (99.99\%). The material was rolled with different amounts of cold reduction $(35 \%, 60 \%$, $80 \%$ and $90 \%$ ) to the same thickness, giving four different starting textures. Fig. 3 shows the crystallite orientation distribution function (CODF) intensities along the squeleton line for these textures. This figure indicates that a strong rolling texture exists in all of the material conditions with relatively similar CODF intensity. However, the intensity of the cube texture decreases as the cold rolling reduction increases from $30 \%$ to $90 \%$. For each sheet, circular blanks were drawn into cups. The flange radii of the drawn cups were measured along the circumference with a computer-controlled noncontacting measuring device. The data at diametrically opposite sides were averaged to exclude error due to eccentricity of the tooling system. More details about the material processing and the deep drawing experiments have been published previously? ${ }^{7}$. Plane stress earing predictions using yield surfaces calculated with the Taylor/Bishop and Hill model, were also given in that work. The radius of the cup after drawing was simply given by:

$$
r_{d}\left(\theta+90^{\circ}\right)=r\left\{1+\frac{1-d}{d} \frac{v_{r}\left(\theta+90^{\circ}\right)}{v_{r}\left(90^{\circ}\right)}\right\}=r\left\{1+\frac{1-d}{d} \frac{\sigma(\theta)}{\sigma\left(0^{\circ}\right)}\right\}
$$

where $r$ is the initial flange radius, $r_{d}$ is the flange radius at a given drawing ratio, $d=r / r_{d}\left(90^{\circ}\right)$, and $\sigma$ the uniaxial or plane strain yield stress in a direction defined by $\theta$. These predictions were found to be in good agreement with the experiments at high rolling reductions ( $80 \%$ and $90 \%$ ), but not for those at low rolling reductions where the 
textures consisted of a mixture of recrystallization and rolling components. Several reasons have been proposed to explain this disagreement.
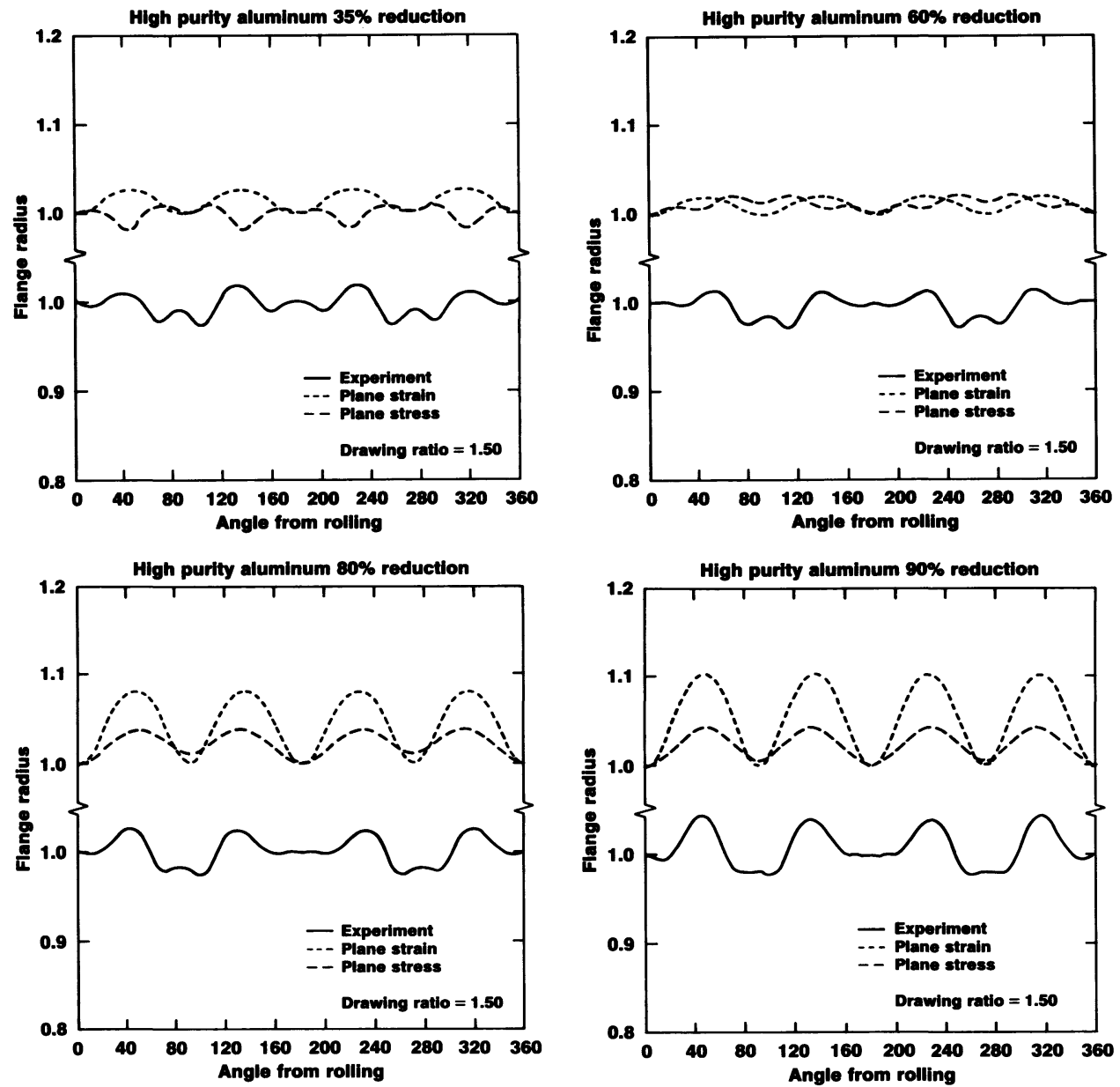

Figure 4. Predicted plane stress and plane strain and experimental flange radius profiles for high purity aluminum sheets with various cold rolling reductions.

The first reason is related to the mathematical description of the CODF using the spherical harmonic series method. With this method only the even coefficients of the series expansion can be calculated and, as a consequence, for some given orientations the calculated intensities are negative, which has no physical meaning. Since the yield surface shape can be influenced by these "ghost values," they have been recalculated with a new CODF computation procedure based on the positivity condition ${ }^{10}$. However, although the new computations somewhat modified the earing profiles they did not significantly change the ear and trough positions.

The second reason proposed to explain the disagreement between earing predictions and experiments for low cold rolling reduction is related to the choice of the strain or stress state assumed in the flange for the calculations. It has been shown previously that plane stress and plane strain earing are different for a single crystal in the cube position. 
In a real cup drawing operation, the stress or strain state in the flange is probably between these two limiting cases. Therefore, it seems reasonable to look at the effect of the deformation mode on earing tendency by computing the earing profile in the two limiting cases.

In the following, material properties have been obtained only with the CODF computation procedure which eliminates ghost peaks. Earing profiles have been computed for high purity aluminum in each of the four material conditions using the plane stress (no blank holder force) or the plane strain (no thickening of the flange) assumptions. The results appear in Fig. 4. For the sheet with $35 \%$ cold rolling reduction, $0^{\circ}$ and $90^{\circ}$ earing is predicted under the plane stress condition whereas, with the plane strain assumption, $45^{\circ}$ earing is predicted. This result seems a bit surprising, since according to the previous section, we would expect $0^{\circ}$ and $90^{\circ}$ plane strain earing for a material with a relatively high amount of cube texture. However, in this example, the texture is quite complicated and it is misleading to rely on conclusions based only on a few ideal texture components. Nevertheless, it appears that plane strain earing is in better agreement with the experiment than plane stress earing, although the small ears occurring at $90^{\circ}$ are not predicted. For the sheet with $60 \%$ cold rolling reduction, it seems that the plane strain prediction again is in better agreement with the position of ears and troughs. Finally, the two last figures correspond to the materials with high amounts of cold rolling reduction. In both plane stress and plane strain cases, ear and trough positions are correctly predicted. The only difference between the two theoretical curves for each material is the earing percentage.

As a general conclusion, the plane strain assumption for the flange behavior successfully explained the major trends for earing of both the single crystal in the cube position and the high purity aluminum sheets with different cold rolling reductions. More accurate predictions might be obtained from more sophisticated models if effects of grain shape, texture evolution and friction were also taken into account.

\section{ACKNOWLEDGEMENTS}

The authors would like to thank Mr. D. J. Lege, Alcoa Laboratories, for valuable comments on the manuscript.

\section{REFERENCES}

1. R. Hill, The Mathematical Theory of Plasticity, (Oxford University Press 1950) p. 328.

2. G.E.G. TUCKER, Acta Met., 9, 275 (1961).

3. G.I. Taylor, J. Inst. Metals, 62, 307 (1938).

4. J.W.F. Bishop and R. Hill, Phil. Mag., 42, 414 and 1298 (1951).

5. M. Grumbach, P. Parnière, L. Roesch and C. Sauzay, Mém. Sci. Rev. Mét., 72, 241 (1975).

6. P. Van Houtte, G. Cauwenberg and E. Aernoudt, Mat. Sci. Eng., 95, 115 (1987).

7. S. Panchanadeeswaran, O. Richmond, L.A. Lalli and W.G. Fricke, Jr., Proc. 8th Int. Conf. Texture of Materials, (TMS 1988), p. 1103.

8. F. Barlat and O. Richmond, Mat. Sci. Eng., 95, 15 (1987).

9. A.D. Rollett, G.R. Canova, and U.F. Kocks, Proc. Conf. Formability and Metallurgical Structure, (TMS 1987), p. 147.

10. H.J. Bunge, Proc. 8th Int. Conf. Texture of Materials, (TMS 1988), p. 69. 